Submitted:

17.05.2021

Accepted: 02.07.2021

Published: 29.11.2021

Keywords mediastinal tumors, ultrasound,

CEUS,

perfusion pattern, diagnosis

\section{Transcutaneous B-mode ultrasound (TUS) and contrast-enhanced ultrasound (CEUS) pattern of mediastinal tumors: a pictorial essay}

\author{
Ehsan Safai Zadeh¹ (D), Christoph Frank Dietrich², Corinna Trenker³, \\ Amjad Alhayri ${ }^{1}$, Christian Görg $^{1}$ (D)
}

${ }^{1}$ Interdisciplinary Centre of Ultrasound Diagnostics; Gastroenterology, Endocrinology, Metabolism and Clinical Infectiology, University Hospital Giessen and Marburg, Philipps University Marburg, Baldingerstraße Marburg, Germany

${ }^{2}$ Department Allgemeine Innere Medizin (DAIM), Kliniken Hirslanden Bern, Beau Site, Salem und Permanence, Switzerland

${ }^{3}$ Haematology, Oncology and Immunology, University Hospital Giessen and Marburg, Philipps University Marburg, Germany

Correspondence: Christian Görg, Interdisciplinary Centre of Ultrasound Diagnostics; Gastroenterology, Endocrinology, Metabolism and Clinical Infectiology, University Hospital Giessen and Marburg, Philipps University Marburg, Baldingerstraße Marburg, Baldingerstraße, 35033, Marburg, Germany; email: christian.goerg@uk-gm.de

DOI: 10.15557/JoU.2021.0056

\begin{abstract}
Sound reflection from bony structures and from air-filled lung parenchyma limits transcutaneous B-mode thoracic ultrasound of the mediastinum. Computed tomography and magnetic resonance imaging of the thorax are the primary overview imaging modalities for mediastinal pathologies. However, pathological processes originating from the mediastinum can be sonographically visualized and evaluated in the presence of displacing or infiltrative growth. The aim of this pictorial essay is to demonstrate contrast-enhanced ultrasound as a method complementing B-mode ultrasound, computed tomography, and magnetic resonance imaging for the evaluation of mediastinal tumors, taking into account the clinical background. The characteristic perfusion patterns of mediastinal pathology are presented.
\end{abstract}

\section{Introduction}

Sound reflection from bony structures and from air-filled lung parenchyma limits transcutaneous B-mode thoracic ultrasound (TUS) of the mediastinum ${ }^{(1)}$. Computed tomography (CT) and magnetic resonance imaging (MRI) of the thorax are the primary imaging modalities used to detect the presence of a mediastinal tumor $(\mathrm{MT})^{(1)}$. However, pathological processes originating from the mediastinum can be sonographically visualized and evaluated in the presence of displacing or infiltrative growth ${ }^{(1)}$. Using contrast-enhanced ultrasound (CEUS), relevant additional information regarding the analysis of perfusion patterns of MTs can be obtained, as this pictorial essay aims to demonstrate. The patient's medical history and clinical symptoms, the location of MTs and, last but not least, histological conformation should always be taken into account when interpreting radiological and sonographic images of MTs ${ }^{(2-4)}$.

Regarding medical history, the patient's age, smoking history, any known benign or malignant systemic disease, and the diagnosis of MT as incidental findings all play a role in the assessment of $\operatorname{MTs}^{(2,3,5)}$. The clinical symptoms may include myasthenic syndrome, B symptoms (fever, weight loss, and night sweats), hoarseness, and superior vena cava syndrome in addition to pressure-induced symptoms ${ }^{(2,6)}$. Regarding the location, characteristic MTs can be diagnosed on ultrasound (US) in the anterior and posterior mediastinum $^{(1)}$. With respect to the TUS patterns of MTs, an assessment of size, border (smooth, irregular), echogenicity 

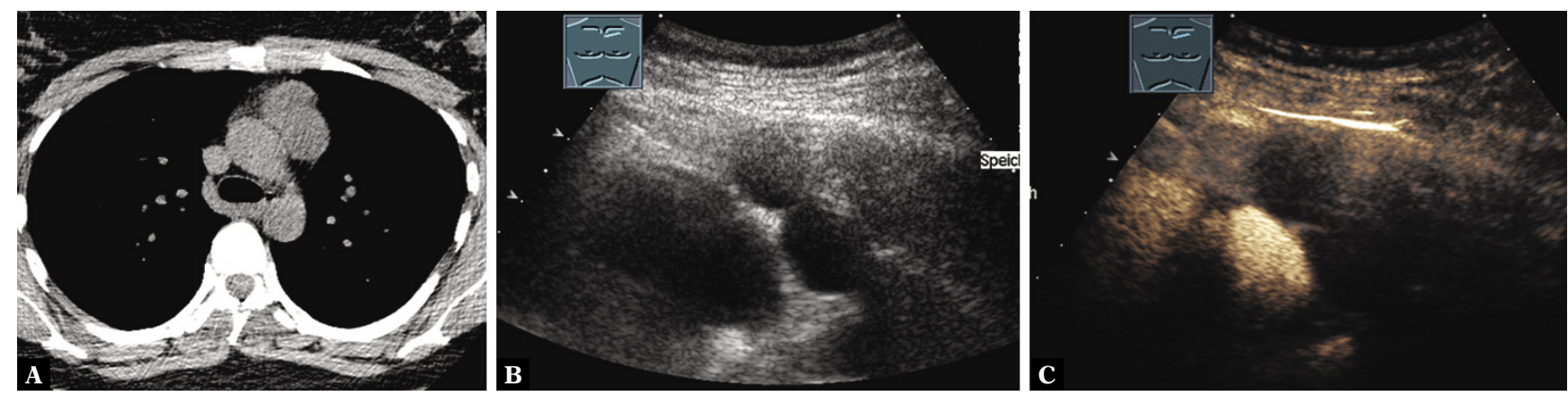

Fig. 1. A 40-year-old female patient with mediastinal tumor in the anterior mediastinum as an incidental finding in computed tomography (A). Thoracic ultrasound indicated anechoic nodes (B), which showed no enhancement on contrast-enhanced ultrasound (C). Mediastinoscopy was performed, and regressive thymoma was histologically confirmed
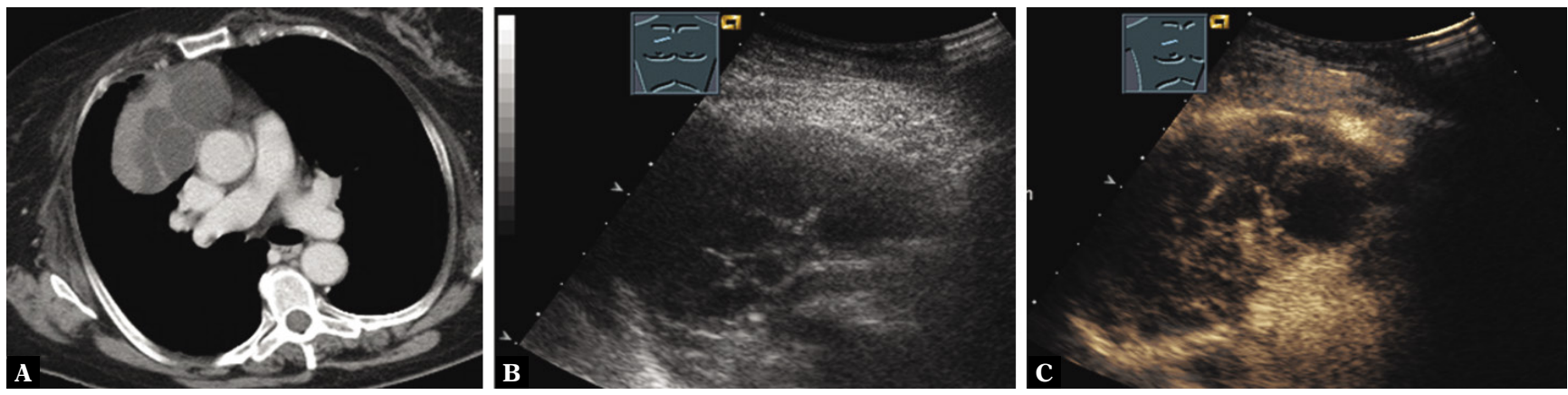

Fig. 2. A 64-year-old female patient with a mediastinal tumor in the anterior mediastinum as an incidental finding on computed tomography (A). Thoracic ultrasound indicated a polyseptated anechoic tumor (B), which showed enhancement of cyst membranes on contrast-enhanced ultrasound (C). Surgical resection was performed. A cystic mediastinal tumor with involved thymus parts was resected. Multilocular cystic hamartoma was histologically confirmed
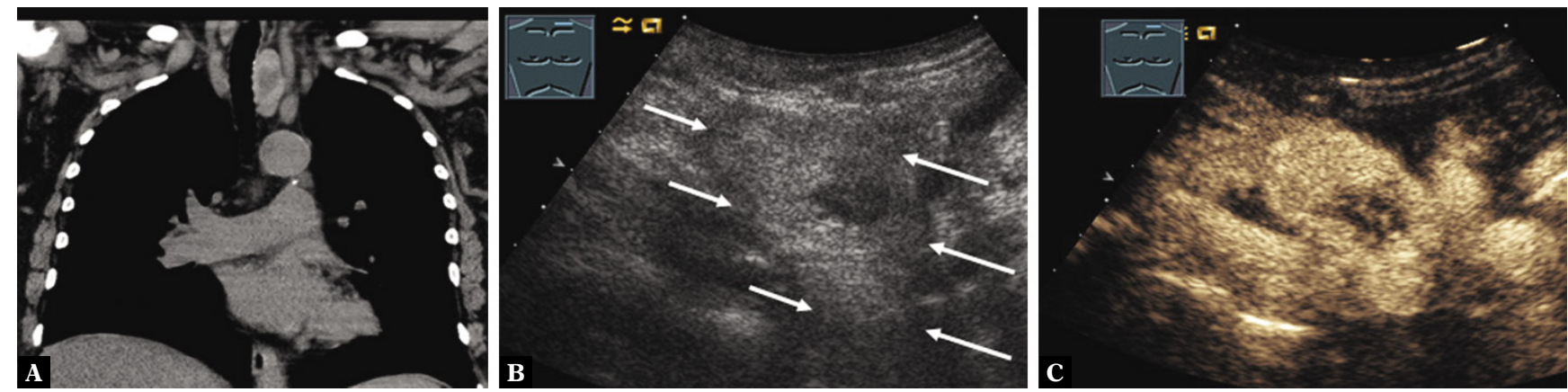

Fig. 3. A 75-year-old female patient with mediastinal tumor in the anterior mediastinum as an incidental finding on computed tomography

(A). Thoracic ultrasound indicated a hypoechoic inhomogeneous tumor (arrows) (B), which showed marked enhancement on contrast-enhanced ultrasound (C). A mediastinal thyroid gland was diagnosed by scintigraphy
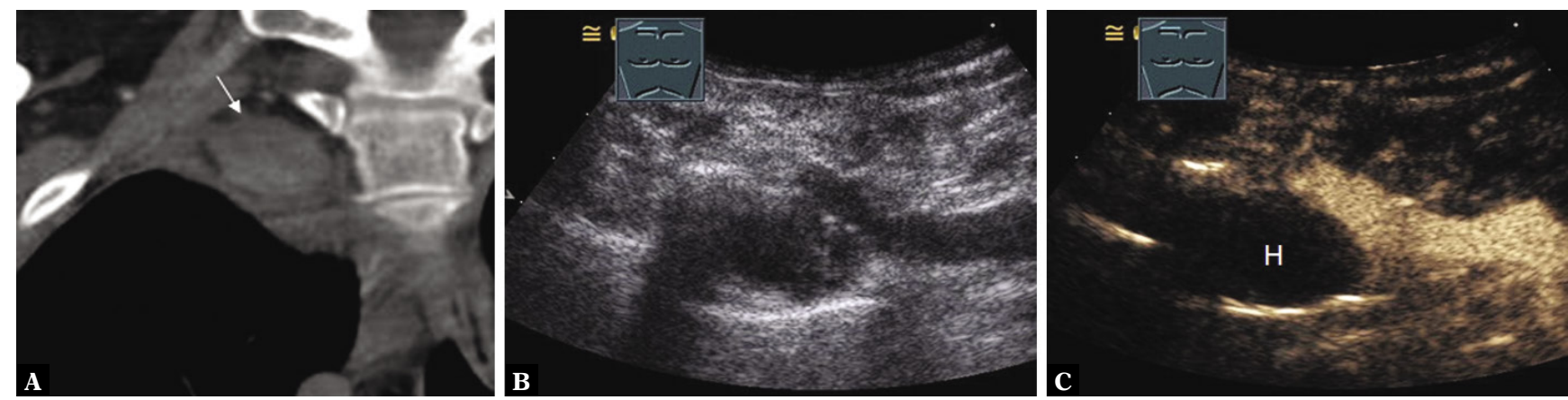

Fig. 4. A 43-year-old female patient admitted for ultrasound-guided biopsy due to mediastinal tumor (arrow) in the anterior mediastinum as an incidental finding on computed tomography after an intensive care stay (A). Thoracic ultrasound indicated a complex hypoechoic tumor $(\mathbf{B})$, which showed no enhancement on contrast-enhanced ultrasound, as in hematoma $(H)(\mathbf{C})$. A biopsy was not performed due to anamnestic central venous catheter. The tumor showed spontaneous regression 

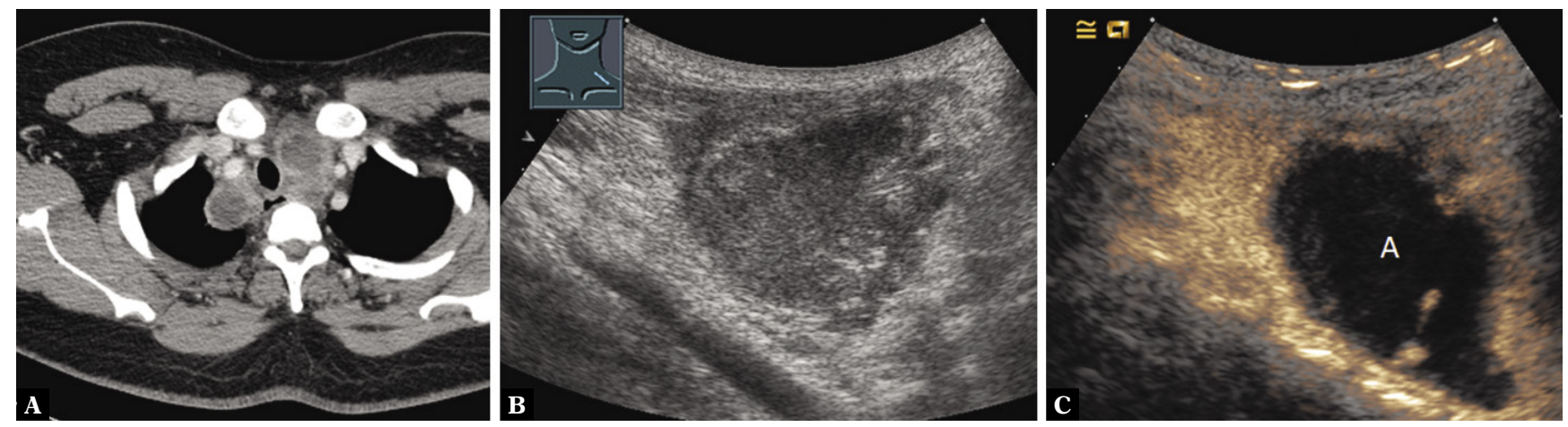

Fig. 5. A 24-year-old male patient with dyspnea and mediastinal tumor (arrow) in the anterior mediastinum as an incidental finding on computed tomography, admitted for ultrasound-guided biopsy (A). Thoracic ultrasound indicated an inhomogeneous, hypoechoic mass (B). On contrast-enhanced ultrasound, large areas showed no enhancement with motion artifacts in real-time examination, as in abscess (A) (C). Ultrasound-guided biopsy was performed. Histologically, granulomatous necrotizing lymphadenitis was found, consistent with lymph node tuberculosis
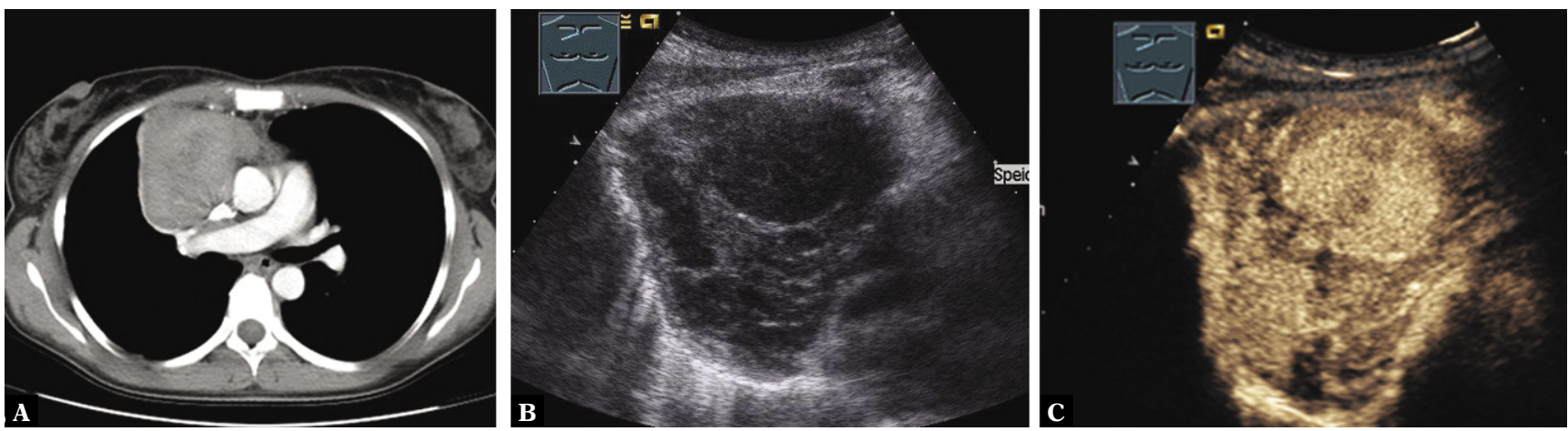

Fig. 6. A 40-year-old female patient with B-symptoms and mediastinal tumor in the anterior mediastinum on computed tomography (A). Thoracic ultrasound indicated a complex hypoechoic tumor $(\mathbf{B})$, which on contrast-enhanced ultrasound showed hyperenhancement, as in malignant lymphoma (C). Ultrasound-guided biopsy was performed, and the diagnosis of Hodgkin lymphoma was confirmed
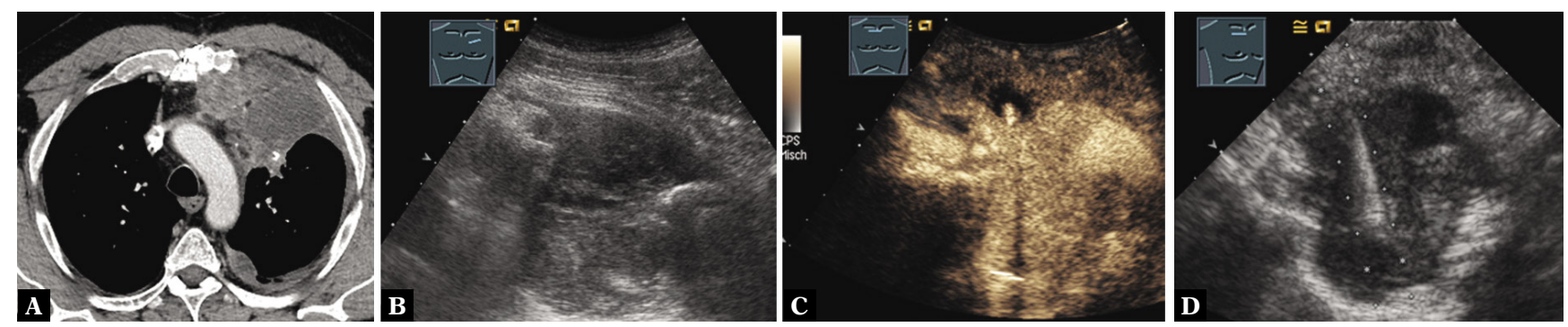

Fig. 7. A 68-year-old male patient with thoracic pressure sensation and tumor in the anterior mediastinum on computed tomography (A). Thoracic ultrasound indicated an inhomogeneous hypoechoic tumor (B), which showed hyperenhancement on contrast-enhanced ultrasound, as in malignant lymphoma (C). Ultrasound-guided biopsy was performed (D), and the diagnosis of diffuse large B-cell lymphoma was histologically confirmed

(anechoic, echogenic, complex), and homogeneity (homogeneous, inhomogeneous) should be performed ${ }^{(1,7)}$. Contrastenhanced ultrasound allows the assessment of contrast enhancement in terms of extent and homogeneity (homogenous, inhomogeneous), and perfused and nonperfused tissue can be reliably differentiated based on the strictly intravascular localized ultrasound contrast medium (CM) (8). In the knowledge that a histology assessment that is relevant for therapy must be performed in the majority of MTs, US-guided biopsy based on the CEUS findings represents a supporting pillar for the evaluation of $\mathrm{MTs}^{(8)}$
This pictorial essay illustrates selected common MTs that were evaluated and documented by a German Society of Ultrasound in Medicine (DEGUM) level 3 qualified examiner (C.G., internal medicine) in the setting of a university hospital with a large oncology department over a 20-year period ${ }^{(9)}$. The CEUS examinations were carried out with a Siemens ACUSON SEQUOIA 512 GI ultrasound scanner (Siemens, Erlangen, Germany) and a 4C1 curved array transducer in $1.5 \mathrm{MHz}$ contrast-specific mode, and a bolus injection of $2.4 \mathrm{ml}$ of SonoVue ${ }^{\circledR}$ (Bracco Imaging S.p.A., Milan) CM via a peripheral 

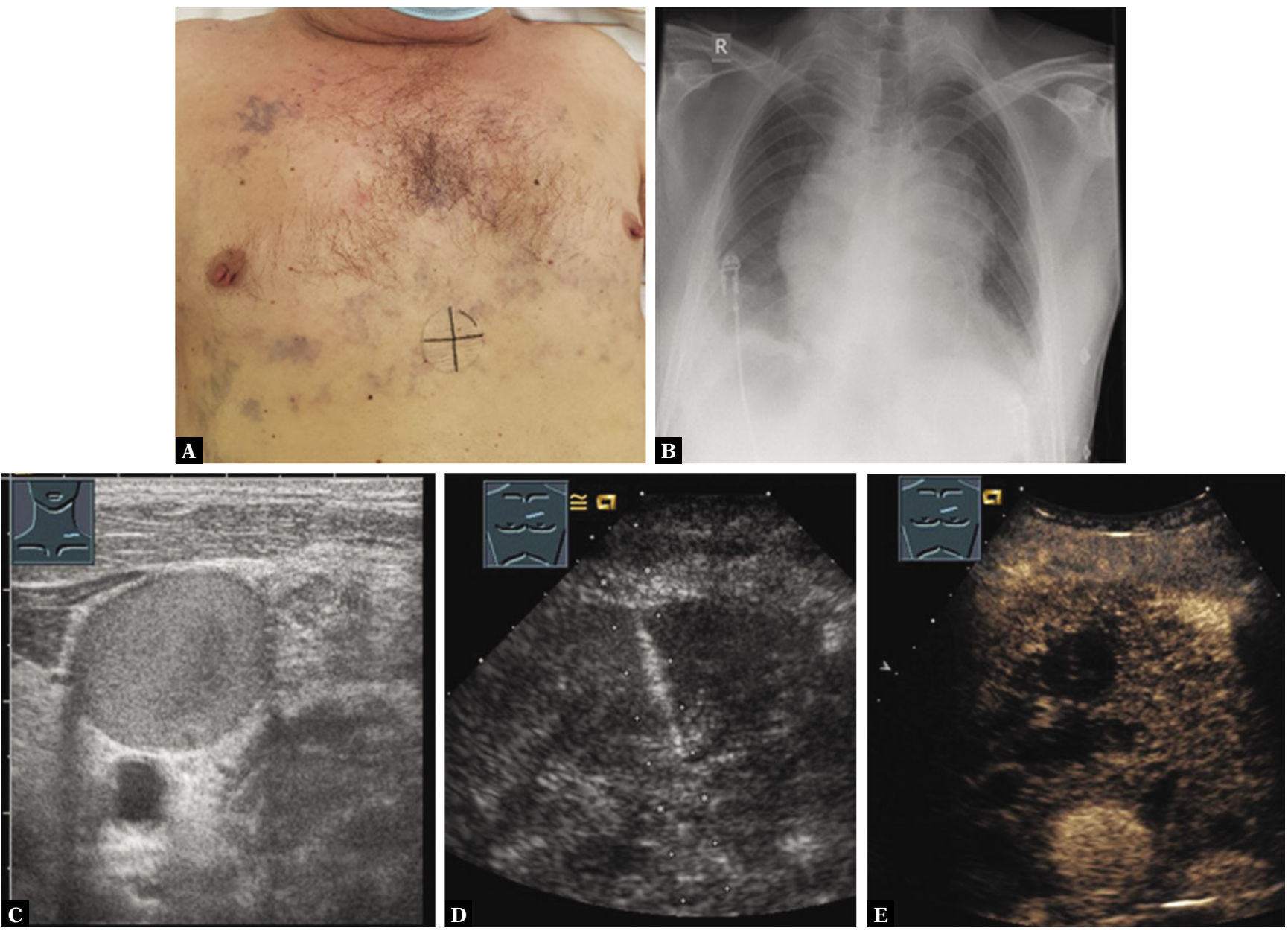

Fig. 8. A 47-year-old male patient with a smoking history and thoracic pressure sensation, increased thoracic cutaneous vessels (A) and mediastinal tumor on chest $x$-ray $(\mathbf{B})$. Thoracic ultrasound revealed echogenic jugular veins with slow flow, as in superior vena cava syndrome (C), and mediastinal inhomogeneous echoic tumor $(\mathbf{D})$, which showed inhomogeneous enhancement with necrosis (N) on contrast-enhanced ultrasound (E). A diagnosis of bronchial carcinoma was confirmed by ultrasound-guided biopsy
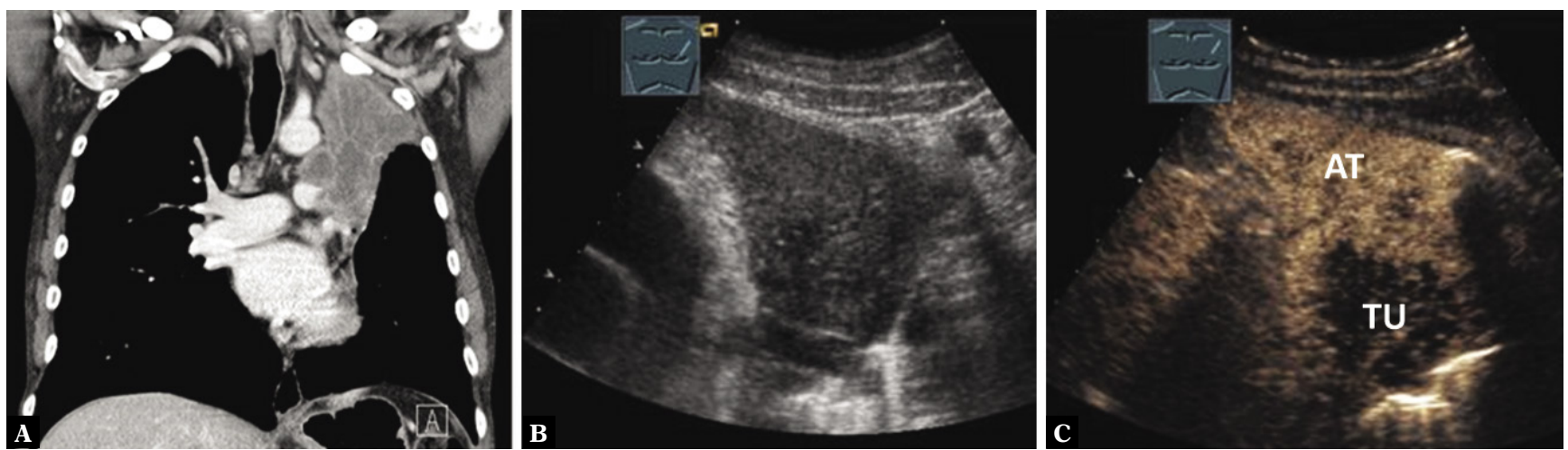

Fig. 9. A 46-year-old male patient with thoracic pressure and mediastinal tumor in the anterior mediastinum on computed tomography (A). Thoracic ultrasound indicated homogeneous low echo consolidation $(\mathbf{B})$, which shows mixed central reduced enhancement on contrast-enhanced ultrasound. Here, the central tumor (TU) demarcates from the atelectasis (AT) (C). Transatelectatic ultrasound-guided biopsy was performed, and the diagnosis of bronchial carcinoma was confirmed histologically

venous access according to the European Federation of Societies for Ultrasound in Medicine and Biology (EFSUMB) guidelines ${ }^{(10)}$. None of the patients had any contraindications for CEUS examination with SonoVue, including hypersensitivity to the ultrasound CM, known right-to-left shunts, severe pulmonary hypertension, adult respiratory distress syndrome (ARDS) according to the HOROWITZ index, and pregnancy or breastfeeding ${ }^{(11)}$. Informed consent for ultrasound examinations was obtained from each patient. 

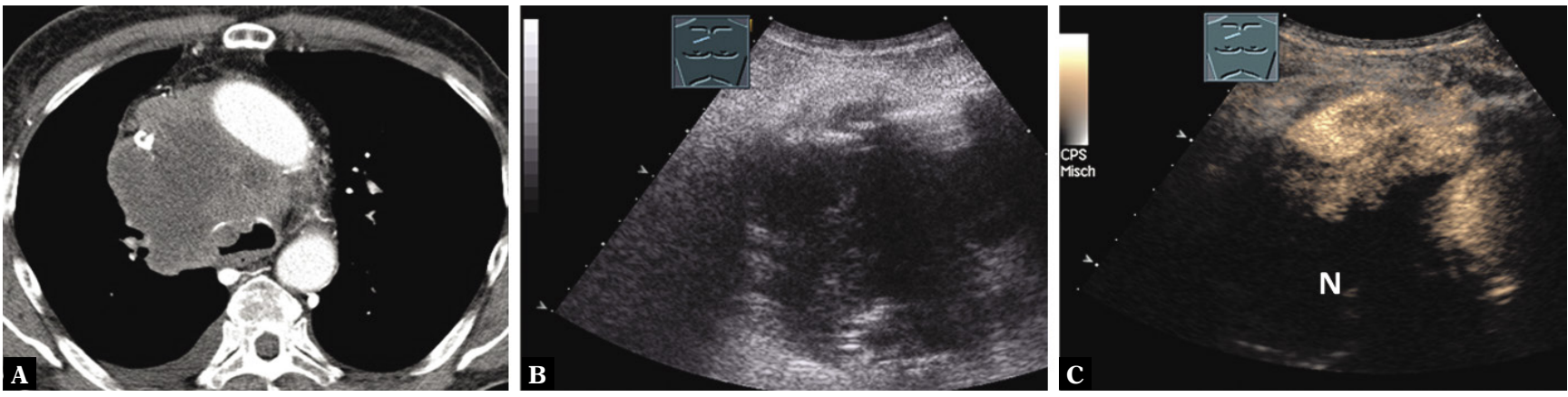

Fig. 10. A 71-year-old male patient with dyspnea and a mass in the anterior mediastinum on computed tomography (A). Thoracic ultrasound indicated a complex hypoechoic tumor $(\mathbf{B})$, which on contrast-enhanced ultrasound showed large areas with lack of enhancement, as in necrosis (N), and only marginal vital tissue (C). Ultrasound-guided-biopsy from perfused tissue was performed, and the diagnosis of bronchial carcinoma was confirmed histologically
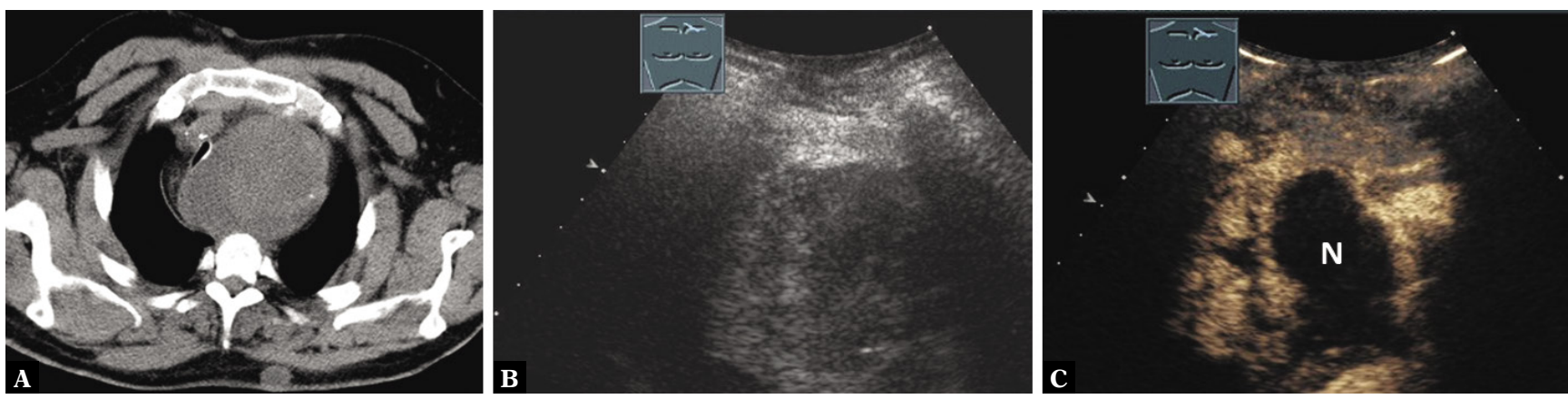

Fig. 11. A 48-year-old male patient with dyspnea and a mass in the anterior mediastinum on computed tomography (A). The patient had a history of a germ cell tumor. Thoracic ultrasound indicated a hypoechoic tumor $(\mathbf{B})$, in which contrast-enhanced ultrasound showed large areas with absent enhancement, as in necrosis $(N)(\mathbf{C})$. Ultrasound-guided biopsy from perfused tissue was performed, and the diagnosis of mediastinal malignant teratoma metastasis was histologically confirmed
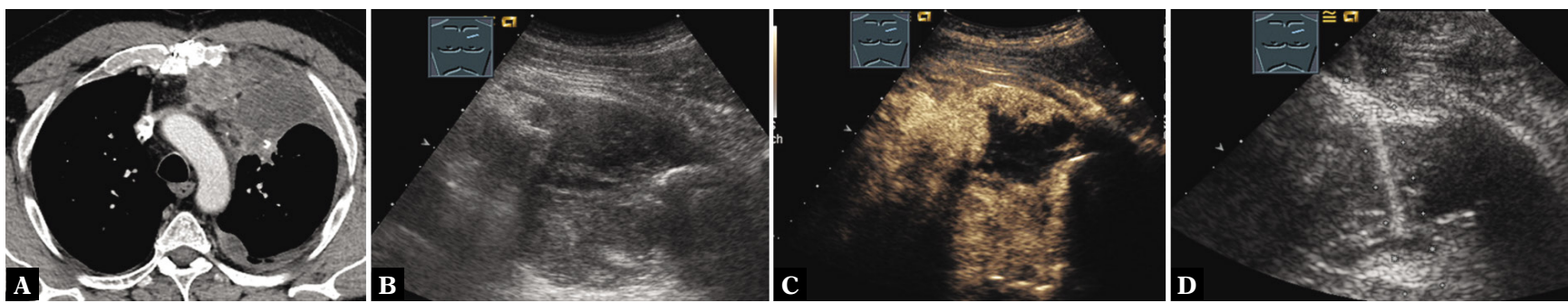

Fig. 12. A 52-year-old male patient with thoracic pressure sensation and mediastinal tumor in the anterior mediastinum on computed tomography (A). An osteosarcoma was known from the medical history. Thoracic ultrasound indicated an inhomogeneous hypoechoic tumor (B), which showed mixed central absent enhancement on contrast-enhanced ultrasound (C). Ultrasound-guided biopsy was performed, and the diagnosis of a mediastinal sarcoma metastasis was histologically confirmed (D)
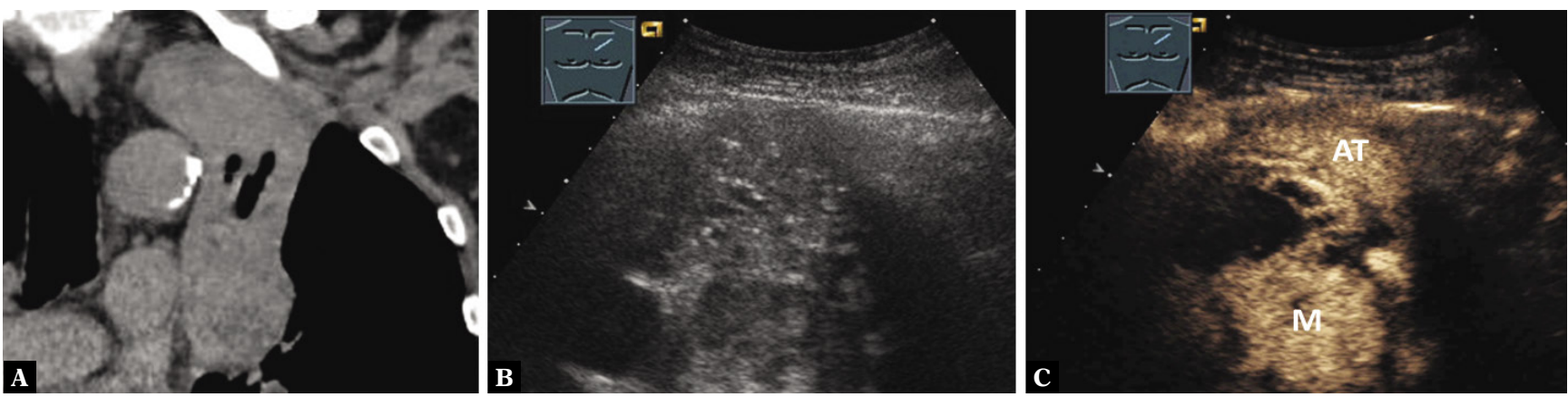

Fig. 13. A 58-year-old male patient with thoracic pressure sensation and a mediastinal tumor in the anterior mediastinum on computer tomography (A). A history of renal carcinoma was known. Thoracic ultrasound indicated a dumbbell-shaped, centrally hypoechoic and peripherally inhomogeneous echogenic tumor (B), which on contrast-enhanced ultrasound shows a marked enhancement centrally and peripherally, similar to that in peripheral atelectasis $(A T)$ and central metastasis $(M)(\mathbf{C})$. The diagnosis of a metastasis from renal cell carcinoma was histologically confirmed 

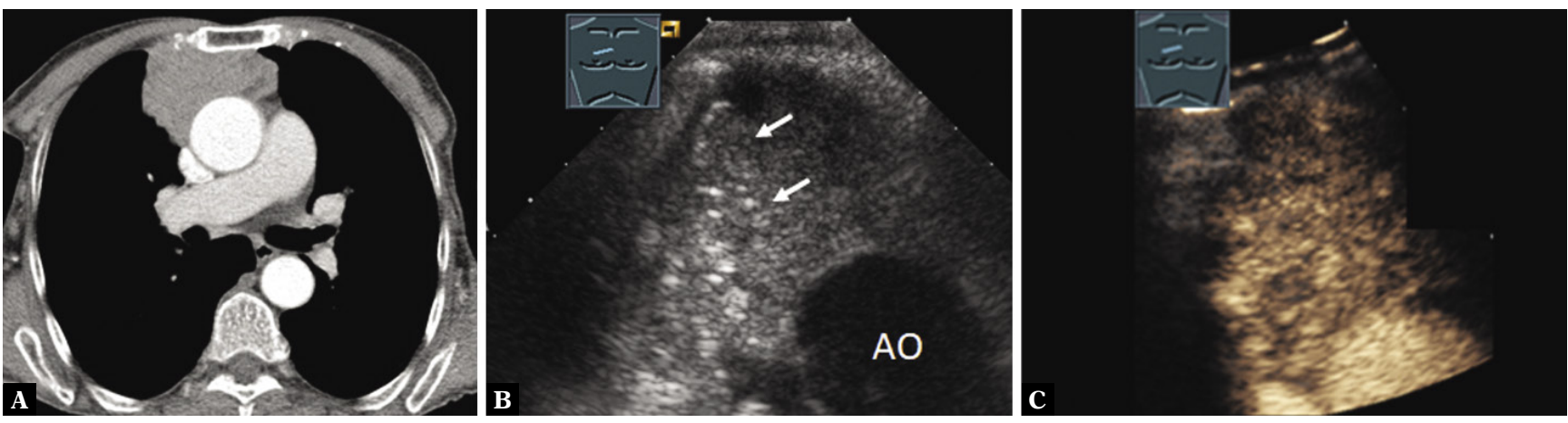

Fig. 14. A 79-year-old female patient with a mediastinal tumor in the anterior mediastinum on computed tomography as an incidental finding (A). Thoracic ultrasound indicated a hypoechoic tumor with localized multiple small star-like reflexogenic lesions (arrows) (AO = aorta) (B); the tumor showed marked enhancement on contrast-enhanced ultrasound (C). Ultrasound-guided biopsy was performed, and the diagnosis of B2 lymphoepithelial thymic carcinoma was histologically confirmed
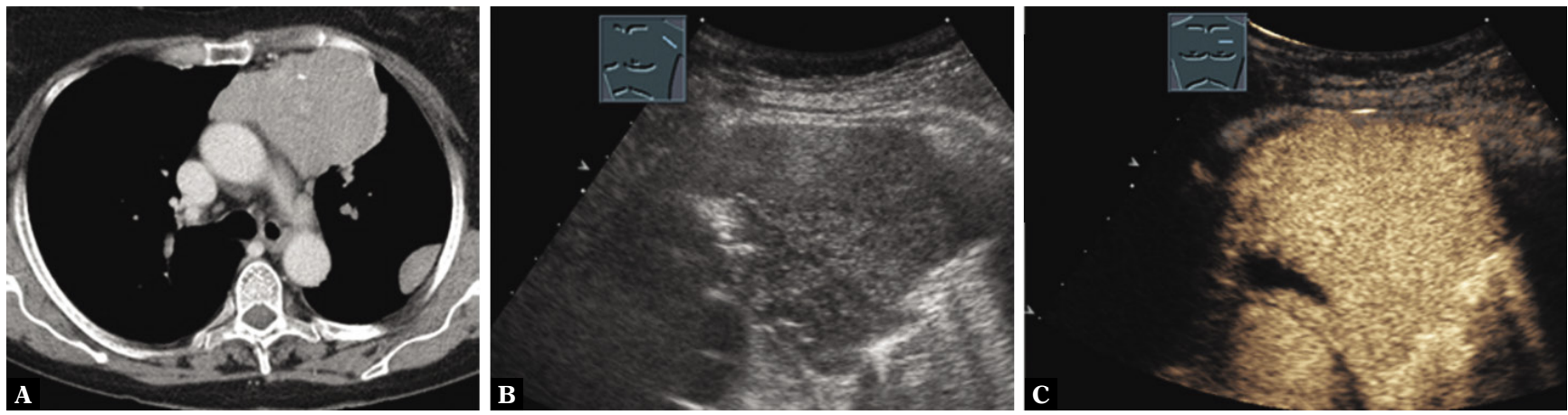

Fig. 15. A 71-year-old female patient with thoracic pressure sensation and a mediastinal tumor in the anterior mediastinum on computed tomography as well as an additional pleural lesion (A). Thoracic ultrasound indicated a hypoechoic lesion (B); the tumor showed marked homogeneous enhancement on contrast-enhanced ultrasound (C). Ultrasound-guided biopsy was performed, and the diagnosis of thymic carcinoma B1 was histologically confirmed
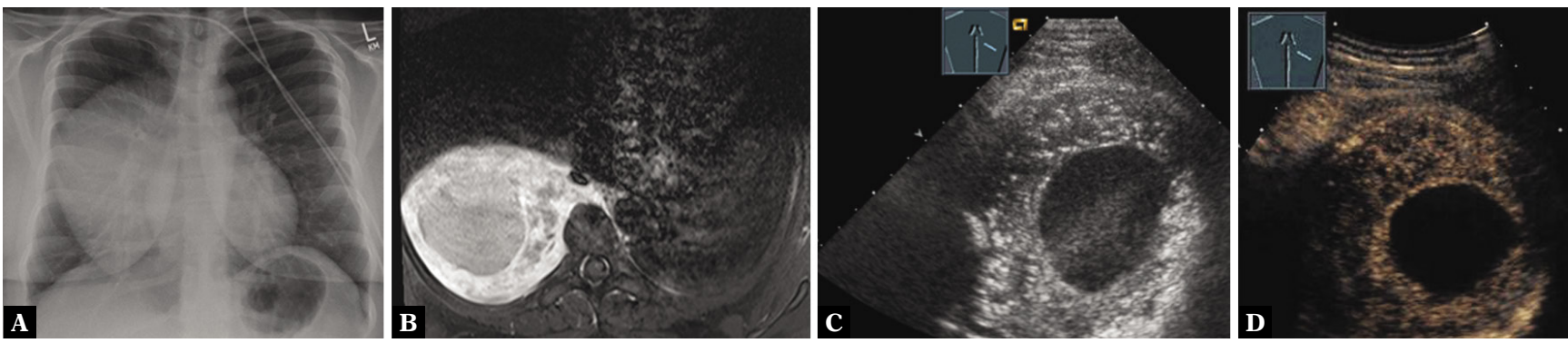

Fig. 16. A 27-year-old male patient with thoracic pressure sensation and a mediastinal tumor in the posterior mediastinum on chest $x$-ray (A). The tumor was visualized by MRI (T2 weighting) (B). Thoracic ultrasound indicated an inhomogeneous hypoechoic tumor, marginally solid with small reflexogenic lesions, centrally anechoic $(\mathbf{C})$, which on contrast-enhanced ultrasound showed mixed centrally absent enhancement and peripheral hypoenhancement (D). Ultrasound-guided biopsy was performed, and the diagnosis of ganglioneuroma was histologically confirmed

\section{Tumors of the anterior mediastinum on contrast-enhanced ultrasound}

Among the primary benign MTs located in the anterior mediastinum, regressive thymoma lesions (Fig. 1) $)^{(1,3,12)}$, mediastinal cysts (Fig. 2) ${ }^{(1,3)}$, mediastinal ectopic thyroid gland (Fig. 3) (3) , post-interventional mediastinal hematoma (Fig. 4) ${ }^{(13)}$, and mediastinal abscess formation (Fig. 5) ${ }^{(14)}$ must be considered.

Primary malignant MTs of the anterior mediastinum include mostly Hodgkin lymphoma (Fig. 6) ${ }^{(1,3)}$, non-Hodgkin lymphoma
(Fig. 7) $)^{(1,3)}$, and primary bronchial carcinoma, which may be manifested with superior vena cava syndrome ${ }^{(15)}$ (Fig. 8), by a poststenotic atelectasis as an "acoustic window"(16) (Fig. 9), or by a tumor with pronounced necrosis (Fig. 10). A definitive diagnosis can be made by US-guided punch biopsy, if necessary ${ }^{(4)}$. Mediastinal metastases are common, and may present as MTs in almost all malignant systemic diseases (Fig. 11 and Fig. 12) $(1,3,17)$. Occasionally, the mediastinum can also be seen here by poststenotic atelectasis (Fig. 13). Malignant thymoma or thymic carcinoma (Fig. 14 and Fig. 15) are markedly less common, and are usually located in the anterior superior mediastinum ${ }^{(18)}$. 

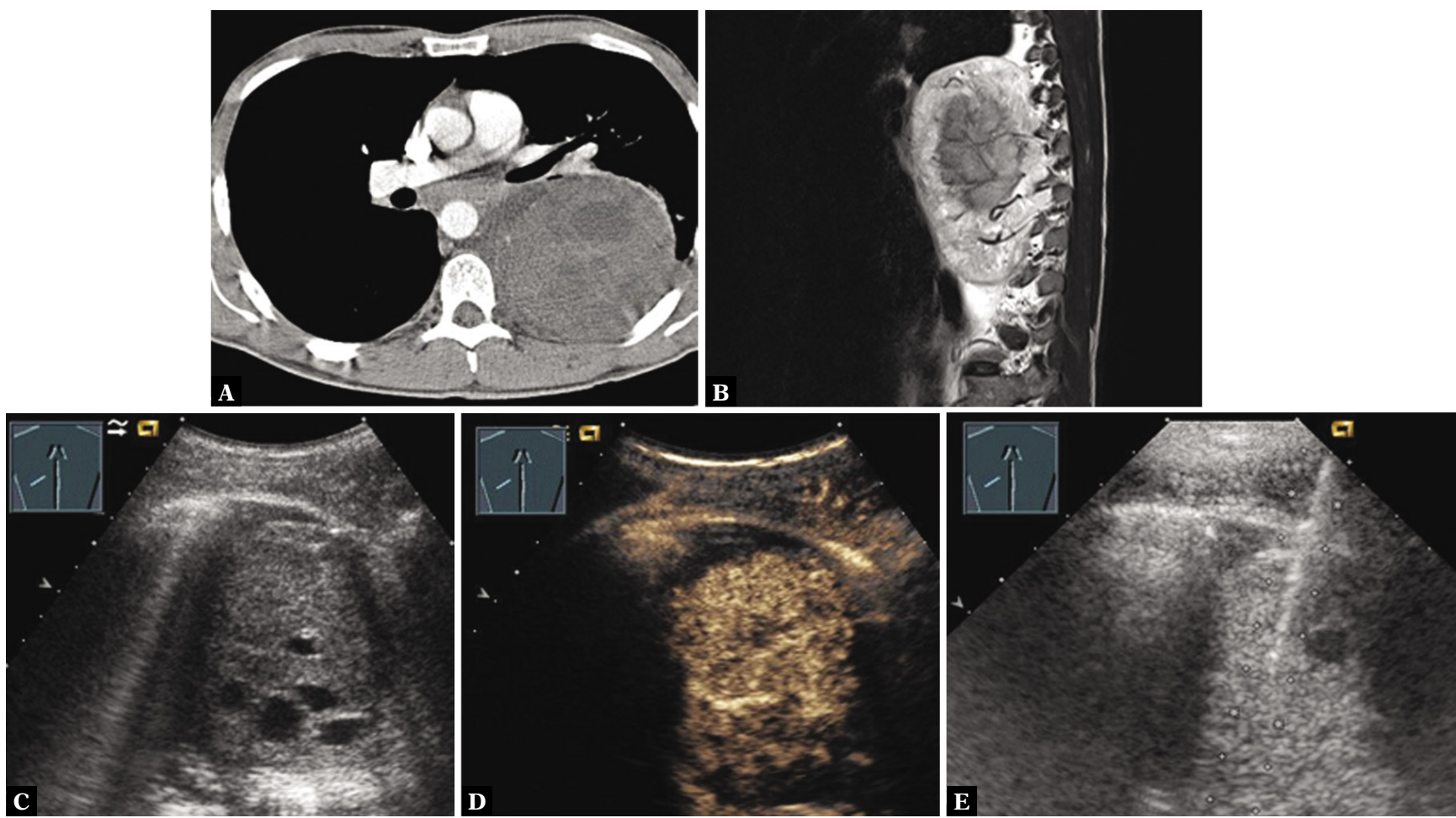

Fig. 17. A 34-year-old male patient with thoracic pressure sensation and a mediastinal tumor in the posterior mediastinum on computed tomography (A). The tumor was visualized by MRI (T2 weighting) (B). Thoracic ultrasound indicated an echogenic tumor with small anechoic lesions (C), which showed inhomogeneous marked enhancement on contrast-enhanced ultrasound (D). Ultrasound-guided biopsy was performed (E), and the diagnosis of schwannoma was histologically confirmed
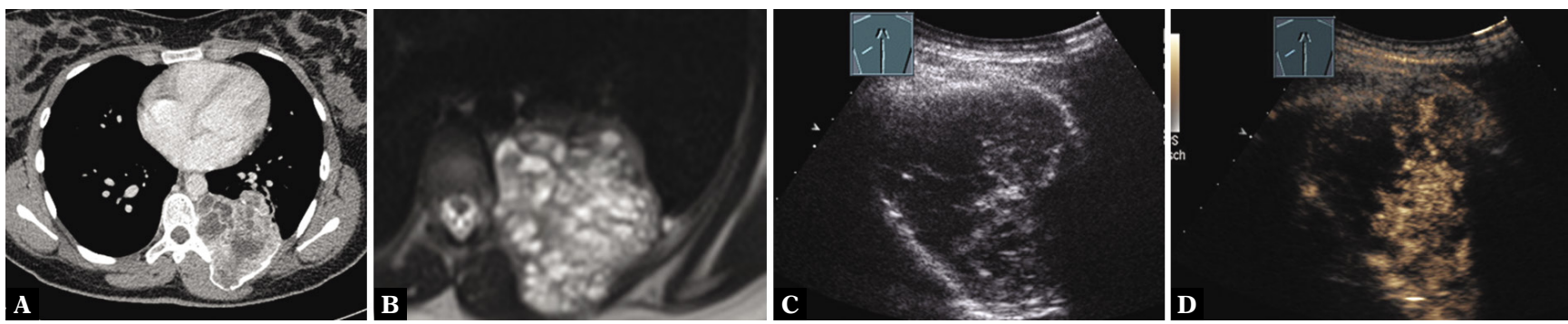

Fig. 18. An 18-year-old male patient with thoracic pressure sensation and a mediastinal tumor in the posterior mediastinum on computed tomography (A). The tumor was visualized by MRI (T2 weighting)(B). Thoracic ultrasound indicated a complex tumor with anechoic areas (C), which on contrast-enhanced ultrasound showed inhomogeneous focal enhancement with areas lacking enhancement (D). Ultrasound-guided biopsy was performed, and the diagnosis of giant cell tumor was histologically confirmed
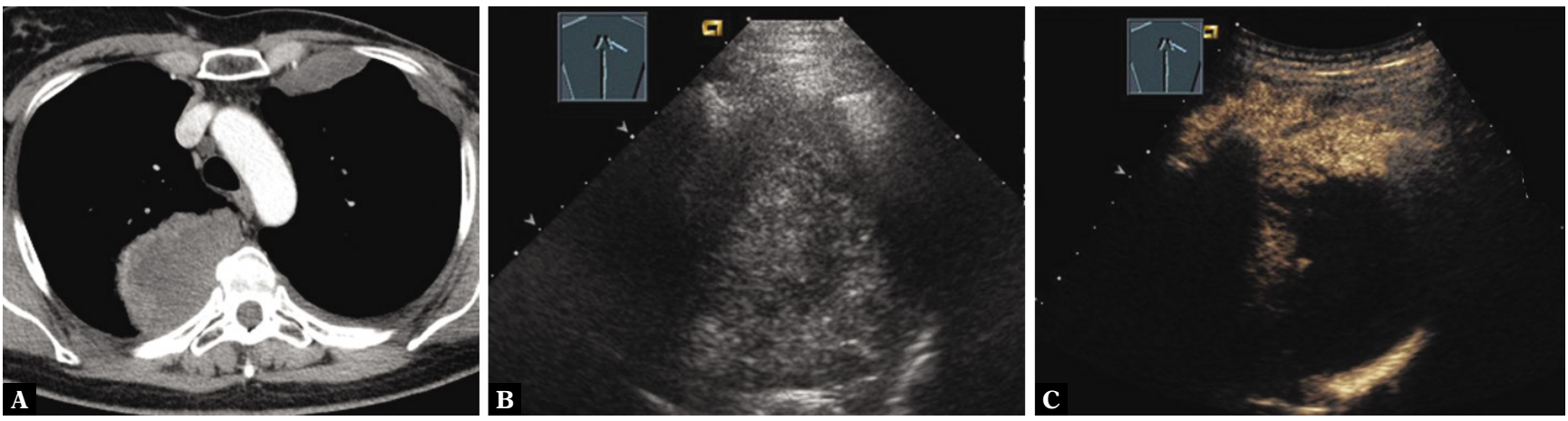

Fig. 19. A 55-year-old female patient with thoracic pressure sensation and a mediastinal tumor in the posterior mediastinum, with an additional ventral pleural lesion on computed tomography (A). A history of malignant phyllodes tumors of the breast was known. Thoracic ultrasound indicated an inhomogeneous hypoechoic tumor (B), which on contrast-enhanced ultrasound showed a mixed central absent enhancement and marginal marked enhancement (C). Ultrasound-guided biopsy was performed, and the diagnosis of a metastasis from a malignant phyloid tumor of the breast was histologically confirmed 


\section{Tumors of the posterior mediastinum on contrast-enhanced ultrasound}

The posterior mediastinum can be viewed sonographically only to a limited extent ${ }^{(19)}$. Solely large MTs will be seen, but they are a rarity in US diagnostics. Here, the more common benign neurogenic tumors, such as ganglioneuroma (Fig. 16)(3) and schwannoma (Fig. 17) (3) $^{(3)}$ well as rarer entities including benign giant cell tumor (Fig. 18), can be confirmed by US-guided punch biopsy ${ }^{(1)}$. Secondary mediastinal metastases in the posterior mediastinum are rarely diagnosed by percutaneous sonography (Fig. 19) ${ }^{(3)}$.

\section{References}

1. Chira RI, Chira A, Mircea PA, Valean S: Mediastinal masses-transthoracic ultrasonography aspects. Medicine (Baltimore) 2017; 96: e9082-e9082.

2. Juanpere S, Cañete N, Ortuño P, Martínez S, Sanchez G, Bernado L: A diagnostic approach to the mediastinal masses. Insights Imaging 2013; 4: 29-52.

3. Diederich S: [Tumoren und pseudotumoren des mediastinums]. Radiologe 2012; 52: 1047-1060.

4. Prosch H, Mathis G, Mostbeck GH: Perkutaner ultraschall in diagnose und staging des bronchialkarzinoms. Ultraschall Med 2008; 29: 466-484.

5. Arnon E, Win T, Israel O, Guralnik L, Ben-Haim S:. Lung and Mediastinal Tumors. In: Strauss HW, Mariani G, Volterrani D, Larson SM (eds.): Nuclear Oncology: From Pathophysiology to Clinical Applications. Springer International Publishing 2016: 1-46.

6. Tannous H, Chikwe J, Yammine MB: Mediastinal masses. In: Docimo Jr S, Pauli EM, (eds.): Clinical Algorithms in General Surgery: A Practical Guide. Springer International Publishing 2019: 39-41.

7. Mathis G: Chest sonography. Springer 2017.

8. Trenker C, Dietrich CF, Holland A, Schulte F, Michel C, Görg C: Mediastinal Masses in Contrast-Enhanced Ultrasound - Retrospective Analysis of 58 Cases. J Ultrasound Med 2021; 40: 1023-1030.

9. Heese F, Görg C: [Diagnostische Wertigkeit einer internistischen Referenzsonographie (DEGUM-Stufe 3)]. Ultraschall Med 2006; 27: 220-224.

10. Sidhu P, Cantisani V, Dietrich CF, Gilja OH, Saftoiu A, Bartels E et al:: The EFSUMB Guidelines and Recommendations for the Clinical Practice of Contrast-Enhanced Ultrasound (CEUS) in Non-Hepatic Applications: Update 2017 (Long Version). Ultraschall Med 2018; 39: e2-e44.

\section{Conflict of interest}

Christian Görg received funding from Bracco Imaging. Bracco Imaging supported CEUS workshops at the University Hospital Marburg.

\section{Acknowledgments}

The work of Dr. Ehsan Safai Zadeh is funded as part of a research grant from the Anneliese Pohl Foundation (Anneliese Pohl Stiftung), and we gratefully acknowledge this support. The chest $x$-ray, MRI, and CT images were kindly provided by Prof. Dr. Andreas H. Mahnken, the Director of the Department of Diagnostic and Interventional Radiology at Marburg University Hospital.

11. Hu C, Feng Y, Huang P, Jin J: Adverse reactions after the use of SonoVue contrast agent: characteristics and nursing care experience. Medicine (Baltimore) 2019; 98: e17745.

12. Furuya K, Isobe K, Sano GO, Kaburaki K, Gocho K, Ishida F et al.: Thymoma exhibiting spontaneous regression in size, pleural effusion and serum cytokeratin fragment level: a case report. Mol Clin Oncol 2015; 3: 1058-1062.

13. Smilowitz NR, Saric M, Attubato MJ, Slater JN: Mediastinal hematoma and tracheal compression following transradial percutaneous coronary intervention. Case Rep Cardiol 2018; 2018: 6790120.

14. von Bartheld M, van der Heijden E, Annema J: Mediastinal abscess formation after EUS-guided FNA: are patients with sarcoidosis at increased risk? Gastrointest Endosc 2012; 75: 1104-1107.

15. Goerdt S, Krengel S, Tenorio S, Tebbe B, Geilen C, Orfanos CE: [Das Vena-cava-superior-Syndrom]. Hautarzt 1997; 48: 122-126.

16. Safai Zadeh E, Görg C, Dietrich CF, Görlach J, Alhyari A, Trenker C: Contrast-enhanced ultrasound for evaluation of pleural effusion: a pictorial essay. J Ultrasound Med 2021.

17. Fritscher-Ravens A, Sriram PV, Bobrowski C, Pforte A, Topalidis T, Krause C et al.: Mediastinal lymphadenopathy in patients with or without previous malignancy: EUS-FNA-based differential cytodiagnosis in 153 patients. Am J Gastroenterol 2000; 95: 2278-2284.

18. Harris K, Elsayegh D, Azab B, Alkaied H, Chalhoub M: Thymoma calcification: is it clinically meaningful? World J Surgical Oncol 2011; 9: 95.

19. Wernecke K, Diederich S: Sonographic features of mediastinal tumors. AJR Am J Roentgenol 1994; 163: 1357-1364. 